\title{
Research on the Professional anxiety of News Editors in the era of Intelligent Media
}

\author{
Yuanke Jin ${ }^{1}$, Yan Liu ${ }^{1 *}$ \\ ${ }^{1}$ School of Humanities, Jiangxi University of Traditional Chinese Medicine, Nan Chang, Jiang Xi, China
}

\begin{abstract}
Intelligence-intensive media are in the ascendant driven by algorithms and data. New technology not only leads news editors into the intelligent era, but also has an impact on them. Artificial intelligence carves up the work of news editors, while virtual reality forces the news editing industry to change, and the new concussion of business format causes the professional anxiety of news editors. Based on the background of media intelligence, this paper analyzed the psychological mechanism of professional anxiety caused by intelligent media to news editors from three aspects: cognition, emotion and motivation, it also put forward the scientific cognitive model, the psychological model of the combination of defense and confrontation, and the efficiency improvement model of multi-agent participation.
\end{abstract}

\section{Introduction}

Intelligent media is a self-strengthening ecosystem based on new technologies such as mobile Internet, big data, virtual reality and human-computer interaction, which realizes the intelligent matching of information and user needs[1]. Intelligent media is widely used in business, games, medical, education and other fields, showing certain advantages in massive information retrieval, complex data collation, automatic compilation and visual information presentation. Intelligent media can complete a series of manual editing work, which means that the professional value of news editors will be weakened, and the resulting sense of urgency makes news editors have professional anxiety.

The publishing industry is an ancient industry, which promotes the spread of human knowledge, ideas and culture for a long time. In recent decades, with the rapid development of Internet technology, the development of artificial intelligence is particularly eye-catching, and a large amount of capital has poured into the industry. Scholars continue to explore the influence of smart media on news editors, but most of them focused on the logical framework of macro industry analysis, such as Peng Lan's macro summary of the connotation, denotation, categories and characteristics of smart media[2]. Yu Guoming analyzed its market prospect motivation from the perspective of industrialization[3]. Few scholars discussed the micro-research of intellectual media on the psychology of news editors, which gives this paper important research significance. This paper analyzed the psychological mechanism of news editors' professional anxiety in the context of intellectual media, expounded the news editors' professional anxiety according to the three levels of industry environment, psychological environment and solution path, and explored the solution that can reduce professional anxiety.

\section{Industry environment analysis}

The change of industry environment directly affects the psychological state of news editors.

\subsection{Artificial Intelligence}

The definition of artificial intelligence is controversial. Some scholars have proposed that artificial intelligence belongs to the computer discipline, trying to explore the essence of "intelligence" and produce an intelligent machine that can respond in a way similar to human intelligence[4]. AI is applied to the field of news editing, which shows that intelligent machines simulate manual editing to complete some tasks. Intelligent machines enter the news editing industry, reshaping the internal operation paradigm of the industry. The development of the Internet has profoundly changed people's way of life, communication and reading habits. We can make two judgments: one is that the information explosion will not stop, and time fragmentation and reading fragmentation will become the norm. In addition, big data allows people's character and preferences to be clearly depicted through clicking, reading, buying and other behaviors, personalized communication and push will only become more and more accurate.

\subsection{Virtual reality}

Virtual reality (VR) is a new technology that constructs a simulation environment based on high simulation system, fully dispatches human senses and realizes users' high- 
quality information experience. On the one hand, it is a backlash to the macro news editing industry. Under the condition of VR new technology, news editors need to bring users into the virtual scene through "Internet + sensor" to make them get a sense of on-the-spot experience, but this also causes users to choose excess space and deviate from the news center of gravity track. In the smart media environment, news editors are liberated from the solidified information and have to devote themselves to the creation of visual content. At the same time, editors should also absorb the opinions of the audience and comprehensively adjust the production direction in the process of interaction. On the other hand, it is the pressure on the individual news editors. The production of VR news requires that in addition to the existing planning, arrangement, proofreading and design skills, news editors should also use VR technology to reasonably control the news content.In recent years, with the development of virtual reality technology, augmented reality technology and mixed reality technology under the background of artificial intelligence, the media began to try to use VR and AR technology to produce "immersive news" to create an "immersive" sense of presence for the audience. The production of "immersive news" is through the use of VR and AR technology in news reports, through collection and recording of news facts, and later technical editing presented on the client side of VR and AR, the user is "directly exposed" to the news scene, realizing the integration of the real scene and the virtual scene, and allowing the user to temporarily "peel off" the environment and place himself in the "reality" of the news reporting environment. So as to satisfy the audience's grasp and objective cognition of the news reporting scenes and facts.

\section{Explore the psychological environment}

Zhang Genjia defines professional anxiety as editors' psychological state of nervousness and fear because they are unable to achieve their work goals or overcome difficulties. From the perspective of mental health, occupational anxiety is essentially a psychological imbalance among editors in the process of value comparison, which is closely related to cognition, psychological expectation, sense of efficacy and other factors.

\subsection{Cognitive block imbalance: cognitive bias leads to occupational anxiety}

The utility of intellectual media depends on the psychological cognition of news editors, and cognition is affected by complex factors, so it is inevitable that there will be cognitive bias. Cognitive

bias refers to the error caused by the cognitive subject to the cognitive object in a specific cognitive situation.

The cognitive barrier between news editors and intellectual media comes from cognitive resources and cognitive styles. First, the cognitive resource theory put forward by Fidel and Garcia expounds the important influence of pressure and cognitive resources on the effectiveness of leadership decision-making. Due to the limitation and influence of inherent cognitive resources, news editors often deviate in the choice of cognitive path of intellectual media, especially in the source of information acquisition. In addition, the accumulation of a large number of wrong information leads to the cognitive deviation of news editors from the reality, coupled with the sudden breaking of career stability by intellectual media, which leads to the disorder of their work rhythm and employment direction. Second, the wrong cognitive style misleads the news editor. Smart media brings a brand-new and unknown media environment, and news editors are prone to mistakes in judgment or subjective and objective cognitive dislocation in such a vague cognitive situation, such as magnifying the ability of machine deep learning or worry about the ethical and moral problems of the machine, and so on. Just as the miscognition emphasized by "halo effect" and "halo effect" in psychology is generalized, cognitive bias is also a kind of wrong cognitive logic to infer the whole from the part.

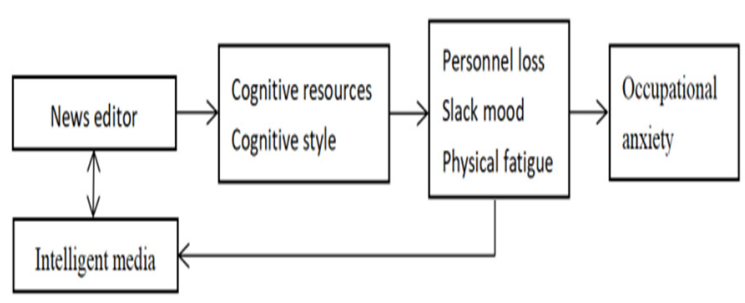

Fig.1. Cognitive bias Model of News Editors.

\subsection{Imbalance of emotional blocks: negative expectations lead to occupational anxiety}

Emotion is the subjective psychological feeling produced by individuals when they encounter stressors, which is affected by psychological expectations. When considering the impact of intellectual media on their careers, editors have a pre-evaluation, and they will make negative predictions according to the current situation of the development of intellectual media, mainly in the following two aspects. New technology leads to the expansion of stressors. Fierce competition among peers and laymen, as well as the invention and application of intelligent devices make it easy for machines to complete editing activities, which extends competitors from people to things. The work intensity is deepened and the psychology is overloaded.

\subsection{Imbalance of motivation blocks: occupational anxiety induced by lack of efficacy}

Motivation is affected by both inducement and internal drive. Self-efficacy belongs to the level of internal drive, which is the intermediary factor to stimulate motivation. Research shows that there is a strong negative correlation between self-efficacy and professional anxiety[6]. When applied to the field of news editors, the higher the sense of self-efficacy, the more confident editors are about their 
own work ability. News editors subconsciously lose confidence in solving new problems, and have a strong anxiety and panic, and then take an evasive way to respond.

\section{Suggestions}

The solution to the professional anxiety of news editors should return to psychology itself and get out of the predicament of anxiety with the help of scientific psychological methods. therefore, it is necessary to put forward targeted suggestions to news editors from three aspects: cognitive model, psychological model and efficiency improvement mode. to help them better adapt to the new environment of the new era.

\subsection{Scientific cognitive model}

The information obtained by news editors based on perception is shallow and extensive irrational judgment, which will inevitably be disturbed by the first cause effect, social stereotype, thinking stereotype and other factors, and fail to understand things in an all-round way. This requires news editors to cultivate a scientific cognitive model and overcome cognitive bias[7]. News editors should establish a correct view of technology and professionalism. The concept of technology needs to be correctly mastered from three aspects. Firstly, news editors should broaden the cognitive channels of intellectual media, establish online group communication, use modern communication tools to collect authoritative intellectual media information interpretation, and accumulate correct cognitive resources. Secondly, news editors should realize that smart media, are still in the "low age" stage, and the lack of abstract abilities such as imagination and innovation is still its fatal weakness. Finally, news editors realize that smart media can help them deal with complicated and time-consuming data, and news editors can devote themselves to the part of the machine blind zone to show their self-worth.

\subsection{Psychological model of the combination of defense and confrontation}

"Defensive" refers to a positive psychological model to relieve anxiety. A survey on editors' defense style and mental health shows that healthy groups are more likely to use mature defense styles to deal with psychological problems than symptom groups[8]. This will be divided into two types: one is communication, that is, news editors interact with professional psychologists to deal with problems with a normal mind by mobilizing various positive psychological elements, and they should also take the initiative to communicate with non-professional personnel to broaden their thinking; second, in the face of the negative impact of intellectual media, news editors can separate their focus from negative thinking and devote themselves to the cultivation of interest in work. "adversarial" means to stimulate the defensive psychology of news editors to fight anxiety. Intelligent technology is a potential threat to news editors, and news editors can regard the pressure caused by this threat as a reverse stimulus to stimulate their potential strength.

\subsection{The efficiency improvement mode of multi- agent participation}

In the actual operation, the difficulty faced by the publishing house is that the publishing house is unable to obtain a large amount of data.

From the terminal point of view, whether online bookstores or physical bookstores, it is difficult to share reader purchase data with publishers; from the supply side, manufacturers do not share data with publishers. As a result, it is difficult for publishers to have enough data to use artificial intelligence to improve decision-making and operations, both on the production side and on the sales side. Multiple subjects include individual, peer and internal management. First of all, to establish an individual effectiveness model with technical training as the core, the company introduces advanced equipment, news editors do hands-on VR and intelligent machines, and gradually accumulates intellectual media experience by setting stage goals for intelligent media learning, so as to enhance the sense of efficacy and control of technology. Secondly, based on the sharing mode of peer-to-peer learning interaction, under the impact of new technology, news editors need to learn successful cases of dealing with technology anxiety among peers or colleagues. We can see that artificial intelligence has made great progress in language through deep learning and other technical means. Although it can not reach the level of artificial practitioners, with the progress of technology, the error rate is getting smaller and smaller. The user experience is getting better and better. This is very valuable for publishers. Finally, news editors and managers need to build the exclusive culture of the enterprise and set up a management model with professional atmosphere as the core.

\section{Conclusion}

In the new era of intellectual media, the news editing industry has experienced a period of transition, which requires news editors to actively transform to fit the new environment, and psychological adjustment is the top priority of the transformation. Because the mental health of news editors is not only related to their own career development, but also to the construction of cultural industry and the effective transmission of spiritual civilization. Therefore, to help news editors get out of the psychological predicament of professional anxiety, it is more necessary to use long-term vision, focus on longterm interests, and promote reform.

\section{References}

1. Quanzhong, G.(2016) The characteristics and construction of intelligent media $[\mathrm{J}]$. News and Writing, (3): 59-62.

2. Lan, P.(2016) Smart Media: future Media Wave: new Media Development trend report [J]. 
International Press, 2016 (11): 7-23.

3. Guoming, Y., Meina, L., Wei, L.(2017) Intelligence: the core logic of future communication mode innovation: also on the basic operation paradigm of artificial intelligence+media $[\mathrm{J}]$. News and Writing,(3): 41-45.

4. Qinying, C.(2017) A brief Analysis of the influence of artificial Intelligence on the Publishing Industry and its Countermeasures [J]. Technology and Publishing, (11): 7-10.

5. Spectrum.(2015) The person in charge of the New York Times headline is a chat robot [EB/OL]. [201508-17].

$\mathrm{Http}: / /$ tech.ifeng.com/a/. 0817/414197380.shtml.

6. Yan, B., Cao, J.(2015) A Review of the Research on Cognitive bias at Home and abroad [J]. Zhengzhou normal Education, (5): 30-Qing.

7. Kai, Yao.(2008) A review of research on self-efficacy: a new trend in the development of organizational behavior [J]. Journal of Management, (5): 463-467.

8. Yanhua, G., Zengxue, Z., Shiguang, S.(2005) The relationship between defense style and mental health of editors of university journals [J]. Hebei Medical Journal of staff and Workers, (9): 28-30. 